\title{
A New Neurological Sign Shows Severity of Carpal Tunnel Syndrome
}

\author{
Sevinç Çelik ${ }^{1}$, Suat Erol Çelik ${ }^{2}$, Canan Bolcu Emir ${ }^{3}$ \\ ${ }^{1}$ Department of Neurology, Fatih Sultan Mehmet Training and Research Hospital, Istanbul, Turkey \\ ${ }^{2}$ Department of Neurosurgery, Okmeydanı Training and Research Hospital, Istanbul, Turkey \\ ${ }^{3}$ Department of Neurology, Okmeydanı Training and Research Hospital, Istanbul, Turkey \\ Email:sevinccelik@yahoo.com, suaterolcelik@yahoo.com, cananemir@hotmail.com
}

Received December 1, 2013; revised January 1, 2014; accepted January 7, 2014

Copyright (C) 2014 Sevinç Çelik et al. This is an open access article distributed under the Creative Commons Attribution License, which permits unrestricted use, distribution, and reproduction in any medium, provided the original work is properly cited. In accordance of the Creative Commons Attribution License all Copyrights @ 2014 are reserved for SCIRP and the owner of the intellectual property Sevinç Çelik et al. All Copyright (C) 2014 are guarded by law and by SCIRP as a guardian.

\section{ABSTRACT}

The objective of this study was prospective comparative determination of both clinical and neurophysiological characteristics of a new pathological reflex in CTS. The authors investigated 300 patients with severe carpal tunnel syndrome undergoing surgery. The 134 patients who showed the sign were allocated as the reflex group. The remaining 166 cases with no sign were named the control group. Two blinded neurology specialists evaluated all patients prior to and after the surgery. Their clinical data, the reflex sign and the electrophysiological results were recorded. The ligament thicknesses during surgery and postoperative changes were also recorded. The reflex group showed exaggerated symptoms with worse electrophysiological results. The reflex group also had thicker median carpal ligament in operative measurements. The difference was significant. The reflex does not disappear easily after surgery and persists up to three months. In the author's opinion, this sign may present a new pathological reflex indicating severe long-lasting nerve compression with the requirement of surgical decompression of the median nerve in CTS subjects.

\section{KEYWORDS}

\section{Carpal Tunnel; Median Nerve; Pathological Reflex; Neurological Sign}

\section{Introduction}

The neurological signs and symptoms of CTS are well known in the daily practice of hand surgeons and neurosurgeons. There is no diagnostic difficulty in the majority of the CTS cases in the preoperative period but confirming of the severity may sometimes be difficult in complicated cases [1]. The neurosurgeon author (SEÇ) has primarily observed and suspected a new neuropathological sign after close investigation of severe CTS cases in the past. The present study was planned to investigate this sign (or reflex) in further detail. This neurological sign (NS) seems to be exaggerated in severe and especially long-term CTS cases. According to the experience of the authors, this abnormality may show a new pathological reflex that indicates a necessity for surgical decompression of the median nerve.
In this study, the authors sought to identify this new NS by evaluating patients in a prospective manner using both electrophysiological and intraoperative findings. The increased chronic pressure of the thickened carpal ligament may exaggerate NS positivity. Ligament measurements from the operated cases were used to confirm the condition.

\section{Patients and Methods}

After the first discovery of the NS during the neurological examination of severe carpal tunnel syndrome patients between 1998 and 2000, this prospective study was planned and performed. The study was initially reviewed by the hospital human studies ethical committee and accepted. At the time of the examination of the patients, the NS was carefully investigated and observed by two blin- 
ded neurology specialists. The duration of the symptoms, the dominant hand and all accompanying diseases were queried. Patients with diabetes or other neurological diseases such as cervical spinal stenosis or disc disease were excluded from study. Electrophysiological studies of the subjects were performed at the other centers before the investigation.

The thickness of the ligament and postoperative clinical recovery duration were also recorded. The results of surgery were evaluated. There were 300 consecutive patients enrolled in the study between 2000 and 2008. All patients underwent x-rays and MRI to rule out bony or other soft tissue abnormality (Figure 1). The 134 patients with the NS were recruited as the NS group. The other 166 patients without the NS were classified as the control group. All patients were operated on by the classical open approach and release of the median carpal ligament. The surgeon was not aware of the presence of the sign when performing the operation. The measurement of the thickness of the carpal ligaments was performed using a surgical caliper (Castroviejo caliper 18000-35, FST Inc., USA) in the middle/thickest portion, at the proximal and distal sections.

The NS appears when the examiner gently taps on the flexor retinaculum with a reflex hammer. A positive result is sudden flexion of the distal interphalangeal joints with flexion of the elbow and shoulder (occasionally) in severe cases (Figure 2). A positive Tinel's sign appears in the majority of the cases on examination of NS but may not be present. The strength of the NS is highly correlated with the severity of the nerve compression in CTS. It appears readily especially in chronic/untreated cases. The authors also validated the presence of the NS and the observation of both examiners was compared for inter-observer error.

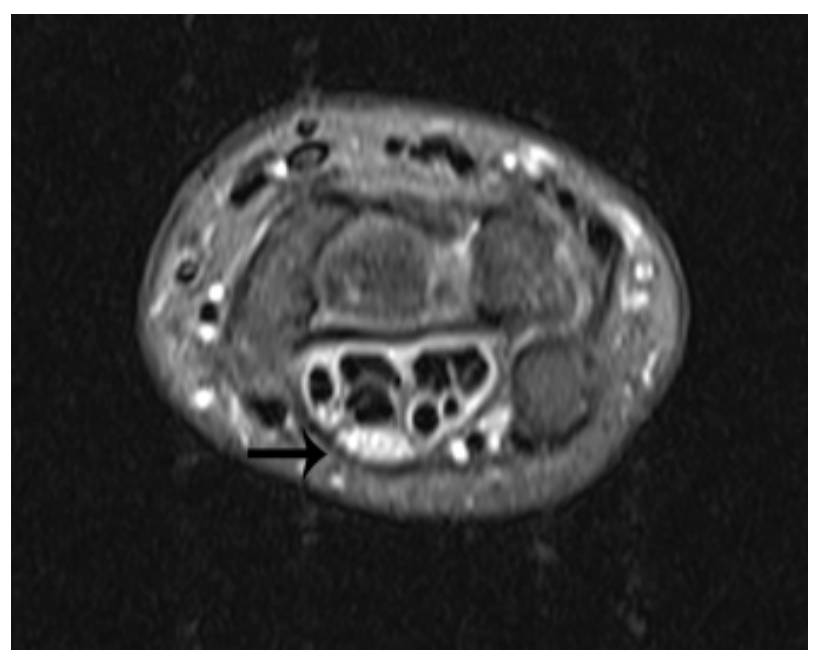

Figure 1. Magnetic resonance imaging studies were used to visualize severe entrapment of the median nerve in carpal tunnel (black arrow).

\section{Statistical Analysis}

Statistical analyses were performed using the $t$ test. Values for $P<0.05$ were considered statistically significant. Results are presented as means \pm standard error.

\section{Results}

A total of 300 patients consisting of 268 women and 32 men were investigated for the presence and severity of the neurological sign. The demographic data and clinical presentation of the patients are displayed in Table 1. NS was clearly present in 134 as determined by two blinded examiners (SÇ and CBE). The NS group showed significantly higher duration of symptoms, hand dominance, increased nerve latencies and lower amplitudes in both sensorial and motor nerve conduction studies (Table 2). The NS group also showed significantly a thickened median carpal ligament in surgical measurements (Table 3). The inter-observer error was not more than $1 \%$ in both groups. The NS appeared to be highly sensitive for the presence of severe CTS.

Postoperatively, the NS did not disappear quickly and generally persisted up to the third postoperative month.

\section{Discussion}

The CTS is a nerve entrapment pathology caused by the chronic compression of the median nerve at the wrist. The reason for this compression is a hypertrophied median carpal ligament $[2,3]$. One percent of the general population is affected by CTS [4,5]. After its first description by Sir James Paget in 1854, the underlying pathology and physical compressive elements became clear with time. Although Pierre Marie and Charles Foix both recommended sectioning the transverse carpal ligament to relieve symptoms in CTS, the first operation was performed by Herbert Galloway and Andrew McKinnon in 1929 [6]. Phalen initially described his transverse incision and discovered painful wrist flexion as a diagnostic sign that was named Phalen sign $[7,8]$. Tinel's test, another diagnostic maneuver, consists of gentle percussion of the median nerve at the wrist. Paresthesias and pain in the distribution of the median nerve indicate a positive response [9,10]. Durkan et al. suggested another examination consisting of direct compression of the median nerve for as long as thirty seconds. The numbness, pain or paresthesias in the hand along the distribution of the median nerve is accepted as a positive response [11,12]. All these tests try to identify pathological compression of the median nerve by the median carpal ligament. Percussion, compression or flexion of the wrist by the examiners may squeeze the nerve, affect its axonal flow, diminish conductivity and exaggerate symptoms.

The test for NS in our observations differs from the previous examination maneuvers. The combination of 


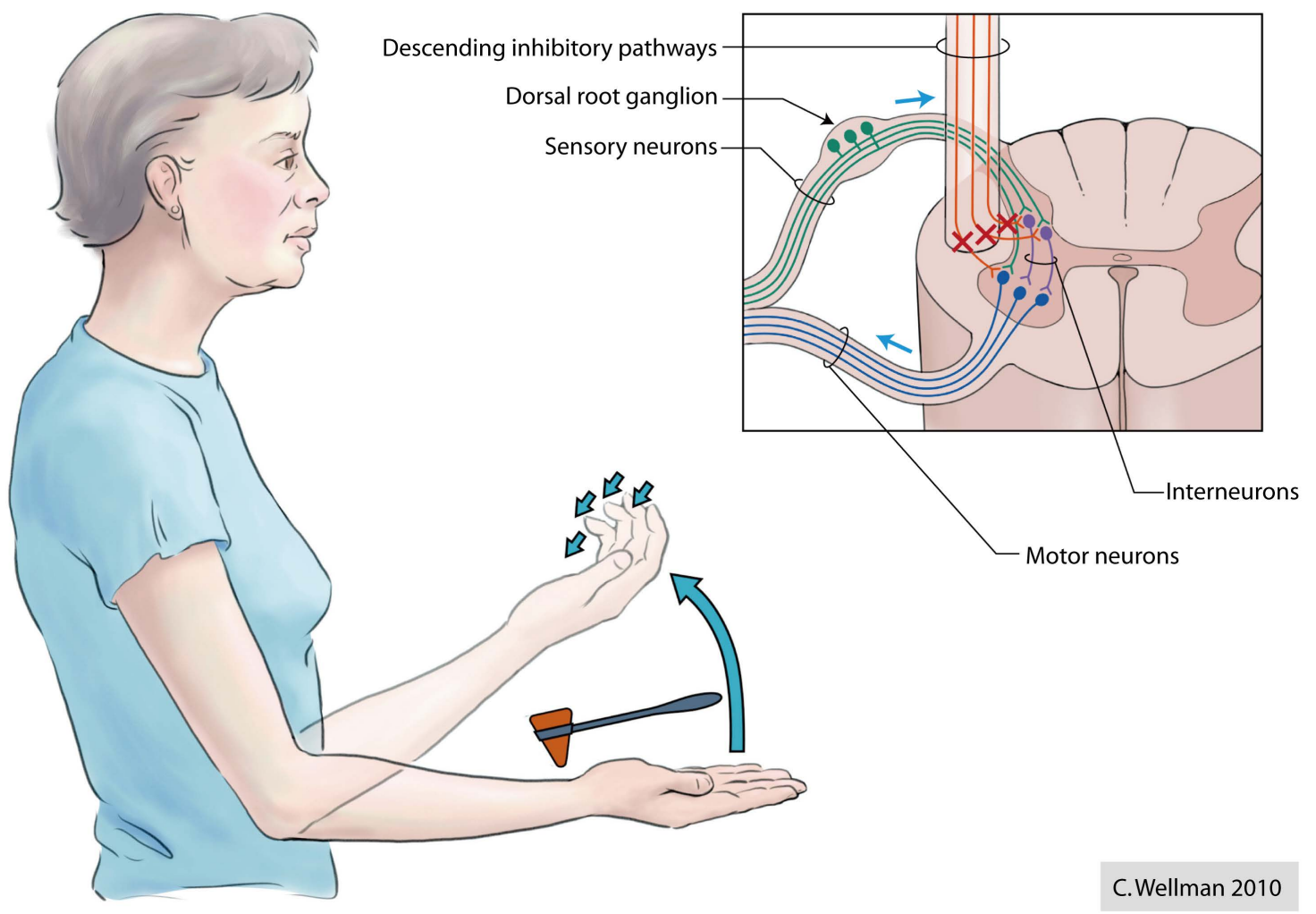

Figure 2. Artist's rendition of the maneuver used to elicit the neurological sign (NS). A positive response is indicated by flexion of the elbow with the fingers. The underlying mechanism of the response is thought to be related to that behind exaggerated deep tendon reflexes via blunting of the descending inhibitory pathways with pathological interneuron activity (upper right).

Table 1. Demographic characteristics of patient groups, data given as mean (SD).

\begin{tabular}{lcc}
\hline Groups & NS (+) $(\mathrm{n}=134)$ & NS $(-)(\mathrm{n}=166)$ \\
\hline Age (years) & $66(17)$ & $59(15)$ \\
Sex (F/M) & $119 / 15$ & $149 / 17$ \\
Hand dominance & $84 \%$ & $82 \%$ \\
Duration of symptoms (months) & $32.6(7.3)^{*}$ & $15(8.6)$ \\
Inter-observer error & $0.74 \%$ & $1.2 \%$ \\
\hline
\end{tabular}

*Significantly higher than other group $(P<0.05)$.

Table 2. Summary of nerve conduction studies of the patient groups.

\begin{tabular}{ccc}
\hline Motor & NS $(+)(\mathrm{n}=134)$ & NS $(-)(\mathrm{n}=166)$ \\
\hline Latency $(\mathrm{m} / \mathrm{sec})$ & $14.2(1 .)^{*}$ & $11.6(2.1)$ \\
Amplitude $(\mathrm{mV})$ & $3.3(1.9)^{*}$ & $5.9(2.3)$ \\
Sensorial & & \\
Latency $(\mathrm{m} / \mathrm{sec})$ & $4.5(2.2)$ & $3.2(2.1)$ \\
Amplitude $(\mu \mathrm{V})$ & $11.9(3.3)^{*}$ & $16.1(3.8)$ \\
\hline
\end{tabular}

*Significantly higher than the other group $(P<0.05)$.
Table 3. Summary of ligament measurements of the patient groups (mm).

\begin{tabular}{lcc}
\hline & NS $(+)(\mathrm{n}=134)$ & NS $(-)(\mathrm{n}=166)$ \\
\hline Proximal third & $7.7(3.5)^{*}$ & $4.3(1.8)$ \\
Middle of the ligament & $12.6(2.2)^{*}$ & $9.3(2.6)$ \\
Distal third & $5.8(2.4)$ & $3.3(2.9)$ \\
\hline
\end{tabular}

*Significantly higher than the other group $(P<0.05)$.

flexion of the shoulder, elbow and fingers emphasizes some degree of hyper excitation and irritability of the spinal cord secondary to chronic median nerve compression.

Various authors have investigated the results of chronic nerve compression from different aspects. The chronic compression in peripheral neuropathies may induce severe irreversible changes in both the spinal cord and peripheral nerves. Girlanda et al. showed the hyper-excitability of the motor neuronal pool in the spinal cord of cubital tunnel patients in 2000. The authors concluded that a peripheral nerve injury might induce pathological rearrangement of nerve circuits at the spinal cord level [13]. After chronic compression or injury, an abnormal barrage of afferent discharge may produce pathological 
neuronal changes to induce a hyperexcitation state in the motoneurons of the spinal cord. Similarly, some structural re-organization of the synaptic connections of the dorsal horn and motoneurons of anterior horn has been shown in the presence of chronic nerve injury. In the presence of these re-innervating changes of the neuronal pool of the spinal cord, the resting state of membrane potentials may switch to a value closer to the threshold for action potential generation [14,15]. Jaberzadeh et al. reached similar findings in their clinical study where they compared chronic CTS patients with normal subjects. They found the flexor carpi radialismotoneuron pool in the spinal cord to be more excitable in chronic CTS patients than in controls [16]. Chronic compressive stress may also induce structural changes in the peripheral nerve. The induction of concurrent apoptosis and proliferation of Schwann cells may lead to neuropathic pain with involvement of reorganized A-delta/A-beta fibers [17,18]. Our new NS may be the result of hyper-excitation stress due to chronic nerve compression. A new pathological reflex circuit via new afferent and efferent pathways may have been created by the rearrangement.

The proposed reflex arc consists of both afferent and efferent pathways with several neural interconnections in the spinal cord. To the author's opinion the main afferent way should be sensory, conveying fibers of the median nerve. The motor jerk response mainly affects the biceps with the elbow flexors, finger flexors and interosseous muscles of the hand. Therefore the possible efferents for this NS may be the median and ulnar nerves of the forearm. The spinal interconnections seem more complex and questionable but have a serious influence on the excitation of C4, C5, C6 and C7 radixes.

Minority of our patients showed NS without Tinel's sign. This condition may be explained by severe nerve dysfunction, inactivity of pain fibers or central ignorance to pain after chronic compression of the nerve.

A single study has previously mentioned a similar response as a motor Tinel's sign in entrapment neuropathies of the cubital tunnel and CTS. This observation included a limited patient size of 30 CTS patients and no surgical results. A muscle jerk with percussion of the median nerve was not visually observed but corresponding myoclonic bursts were demonstrated on electromyographic (EMG) records of the effected muscles in 15 patients [19]. We cannot describe our NS as a motor Tinel's sign only as the motor response shows interactions of both proximal and distal muscles of the forearm, arm and sometimes the shoulder. The findings of the present study clearly support spinal excitation phenomena after severe chronic compression due to peripheral nerve entrapment in CTS. This excitation and pathological synaptic formation probably increase the severity depending on the time and severity of compression. To the author's opinion this NS is a new pathological reflex indicating severe and long-term compression with obvious requirement of surgical decompression of the median nerve in CTS subjects.

\section{REFERENCES}

[1] J. G. Jarvik, B. A. Comstock, P. J. Heagerty, et al., "Magnetic Resonance Imaging Compared with Electrodiagnostic Studies in Patients with Suspected Carpal Tunnel Syndrome. Predicting Symptoms, Function and Surgical Benefit at 1 Year,” Journal of Neurosurgery, Vol. 108, No. 3, 2008, pp. 541-550.

[2] C. A. Pacek, M. Chakan, R. J. Goitz, et al., "Morphological Analysis of the Transverse Carpal Ligament," Hand, Vol. 5, No. 2, 2010, pp. 135-140.

[3] R. C. Tanzer, "The Carpal Tunnel Syndrome. A Clinical and Anatomical Study," The Journal of Bone \& Joint Surgery, Vol. 41, No. 4, 1959, pp. 626-634.

[4] I. Atroshi, C. Gummesson, R. Johnsson, et al., "Prevalence of Carpal Tunnel Syndrome in a General Population," JAMA, Vol. 282, No. 2, 1999, pp. 153-158.

[5] M. Mondelli, F. Giannini and M. Giacchi, "Carpal Tunnel Syndrome Incidence in a General Population,” Neurology, Vol. 58, No. 2, 2002, pp. 289-294.

[6] P. C. Armadio, “The First Carpal Tunnel Release,” Journal of Hand Surgery, Vol. 20, No. 1, 1995, pp. 40-41.

[7] G. S. Phalen, "Spontaneous Compression of the Median Nerve at the Wrist," The Journal of the American Medical Association, Vol. 145, No. 15, 1951, pp. 1128-1133.

[8] G. S. Phalen, "The Carpal-Tunnel Syndrome. Clinical Evaluation of 598 Hands,” Clinical Orthopaedics, Vol. 83, No. 1, 1972, pp. 29-40.

[9] A. W. Siegmeth and J. A. Hopkinson-Woolley, "Standard Open Decompression in Carpal Tunnel Syndrome Compared with a Modified Open Technique Preserving the Superficial Skin Nerves. A Prospective Randomized Study," Journal of Hand Surgery, Vol. 31A, No. 9, 2006, pp. 1483-1489.

[10] J. D. Steward and A. Eisen, “Tinel's Sign and the Carpal Tunnel Syndrome,” British Medical Journal, Vol. 2, No. 6145, 1978, pp. 1125-1126.

[11] J. A. Durkan and H. River, “A New Diagnostic Test for Carpal Tunnel Syndrome,” The Journal of Bone \& Joint Surgery, Vol. 73, No. 4, 1991, pp. 535-538.

[12] T. J. Madhav, P. To and P. J. Stern, “The Palmar Fat Pad Is a Reliable Intraoperative Landmark during Carpal Tunnel Release," Journal of Hand Surgery, Vol. 34A, No. 7, 2009, pp. 1204-1209.

[13] P. Girlanda, A. Quartarone, F. Battaglia, et al., "Changes in Spinal Cord Excitability in Patients Affected by Ulnar Neuropathy,” Neurology, Vol. 55, No. 7, 2000, pp. 975978.

[14] R. Dubner and A. Basbaum, "Spinal Cord Plasticity Following Tissue or Nerve Injury,” In: P. Wall and W. Melzack, Eds., Textbook of Pain, Edinburg Churchill Livingstone, 1994, pp. 243-257. 
[15] B. Javan and E. P. Zehr, "Short-Term Plasticity of Spinal Reflex Excitability Induced by rhythmic Arm Movement," Journal of Neurophysiology, Vol. 99, No. 4, 2008, pp. 2000-2005.

[16] S. Jaberzadeh and S. Scutter, "Flexor Carpi Radialismotoneuron Pool in Subjects with Chronic Carpal Tunnel Syndrome Is More Excitable than Matched Control Subjects,” Manual Therapy, Vol. 11, No. 1, 2006, pp. 22-27.

[17] R. Gupta and O. Steward, "Chronic Nerve Compression Induces Concurrent Apoptosis and Proliferation of Schwann Cells,” Journal of Comparative Neurology, Vol.
461, No. 2, 2003, pp. 174-186.

[18] A. Truini, L. Padua, A. Biasiotta, et al., "Differential Involvement of A-Delta and A-Beta Fibres in Neuropathic Pain Related to Carpal Tunnel Syndrome,” Pain, Vol. 145, No. 1-2, 2009, pp. 105-109. http://dx.doi.org/10.1016/j.pain.2009.05.023

[19] P. Montagna and R. Liguori, “The Motor Tinnel Sign; a Useful Sign in Entrapment Neuropathy,” Muscle Nerve, Vol. 23, No. 6, 2000, pp. 976-978. 